\title{
A MODEL FOR THE GALACTIC POPULATION OF SUPERSOFT X-RAY SOURCES
}

\author{
L. YUNGELSON ${ }^{1}$, A. TUTUKOV ${ }^{1}$, A. FEDOROVA ${ }^{1}$, \\ M. LIVIO $^{2}$, J. W. TRURAN ${ }^{3}$ \\ 1. Institute of Astronomy of the Russian Academy of Sciences, \\ 48 Pyatnitskaya Str., 109017 Moscow, Russia \\ 2. Space Telescope Science Institute, 3700 San Martin Drive, \\ Baltimore, MD 21218, USA \\ 3. Department of Astronomy and Astrophysics, Enrico Fermi \\ Institute of Physics, University of Chicago, \\ 5640 S. Ellis Avenue, Chicago, IL 60637, USA
}

\begin{abstract}
Three major sub-populations of Galactic supersoft X-ray sources may exist: semi-detached binaries with main sequence or subgiant donors, and symbiotic binaries ( $\sim 550, \sim 460$, and $\sim 600$ objects, respectively). Each group contains both permanent and transient sources. The intrinsic and interstellar absorptions reduce the number of observable sources to $\sim 25$. We derive the distributions of the sources over orbital periods, masses of the components, and 'on'-times. The rate at which white dwarfs in Galactic binaries reach $M_{\mathrm{Ch}}$ is $\sim 310^{-5} \mathrm{yr}^{-1}$. The rate of He-shell detonations which may lead to supernovae may be up to $310^{-4} \mathrm{yr}^{-1}$.
\end{abstract}

\section{Discussion}

The common model of supersoft X-ray sources (hereafter SSS) relates the origin of their X-ray emission to steady hydrogen (van den Heuvel et al. 1992) or helium (Iben \& Tutukov 1994) burning at the surface of accreting white dwarfs (WDs). By means of the population synthesis code for binary stars (e.g. Yungelson et al. 1995) we generate a population of systems in which accretion of $\mathrm{H}$ or $\mathrm{He}$ onto $\mathrm{CO}$ or $\mathrm{ONe}$ white dwarfs is possible via Roche lobe overflow or a stellar wind. Following the mass exchange history for each potential SSS (including the secular evolution of accretor mass), we estimate the number of SSS and their properties. Different to earlier

417

A. Evans and J. H. Wood (eds.), Cataclysmic Variables and Related Objects, 417-420.

() 1996 Kluwer Academic Publishers. Printed in the Netherlands. 
TABLE 1. Numbers of supersoft X-ray sources and their parent systems

\begin{tabular}{|c|c|c|c|c|c|c|}
\hline & $\mathrm{CVs}$ & Algols & $\begin{array}{c}\text { Symbiotic } \\
\text { stars }\end{array}$ & $\begin{array}{c}\text { Double } \\
\text { degenerates }\end{array}$ & $\begin{array}{l}\text { Helium } \\
\text { Algols }\end{array}$ & $\mathrm{PNNi}$ \\
\hline \multicolumn{7}{|c|}{ Parent Population } \\
\hline Birthrate, $\mathrm{yr}^{-1}$ & 0.0039 & 0.0006 & 0.047 & 0.013 & 0.0009 & 0.11 \\
\hline Total number & $4.110^{6}$ & $2.410^{5}$ & $1.610^{3}$ & $1.410^{8}$ & 200 & 36 \\
\hline Novae, $\mathrm{yr}^{-1}$ & 20 & 10 & 1.4 & - & - & - \\
\hline Permanent SSS & 134 & 402 & 445 & 70 & $\leq 200$ & 35 \\
\hline Transient SSS & 412 & 59 & 162 & - & - & - \\
\hline \multicolumn{7}{|c|}{ Number of sources with correction for intrinsic absorption by spherical shell } \\
\hline Permanent SSS & 134 & 402 & 0 & & & \\
\hline Transient SSS & 380 & 45 & 7 & & & \\
\hline \multicolumn{7}{|c|}{ Number of 'detectable' sources } \\
\hline Novae, $\mathrm{yr}^{-1}$ & 0.7 & 0.3 & 0.1 & - & - & - \\
\hline $\begin{array}{l}\text { Permanent SSS } \\
(r \leq 2 \mathrm{kpc})\end{array}$ & 2 & 7 & 0 & 1 & $\leq 4$ & $\leq 1$ \\
\hline $\begin{array}{l}\text { Transient SSS } \\
(V \leq 8 \mathrm{mag})\end{array}$ & 8 & 1 & 7 & - & - & - \\
\hline
\end{tabular}

models of SSS, we allow for both stable and unstable nuclear burning of the accreted matter. Subsequently, we consider selection effects and thus obtain the properties of the 'observed' population of SSS. We shall refer to the three main groups of SSS, for brevity, as CVs (cataclysmic variables), Algols (systems with subgiant donors), and SyS (symbiotic systems).

Hydrogen burns stably if $\dot{M}_{\text {acc }}$ exceeds a certain limit. WDs accreting below the stability limit experience hydrogen burning flashes. Once the WD undergoes a flash it loses material, in nova events, via an optically thick wind and/or a common envelope. After the mass of the $\mathrm{H}$-rich layer of the WD decreases below a certain limit, the WD settles in the region of the HR diagram which corresponds to stable burning, and there most of the remaining $\mathrm{H}$ is consumed. Stably burning WDs have $T_{\text {eff }} \gtrsim 250000 \mathrm{~K}$ if $M_{\mathrm{wd}} \gtrsim 0.54 \mathrm{M}_{\odot}$ (Iben \& Tutukov 1989). Hence, all the $\mathrm{CO}$ or $\mathrm{ONe}$ WD accretors may be candidate SSS. During this stage of evolution, the bolometric luminosity is $L \gtrsim 510^{37} \mathrm{erg} \mathrm{s}^{-1}$. For the estimate of the time during which the post-nova WD still emits in the supersoft X-ray range, we use the time it takes the WD to decline by $3 \mathrm{mag}$ in its bolometric luminosity from the Prialnik \& Kovetz (1995) grid of nova models. This estimate gives probably the upper bound of lifetimes.

There are 'permanent' sources, in which $H$ burns steadily, and 

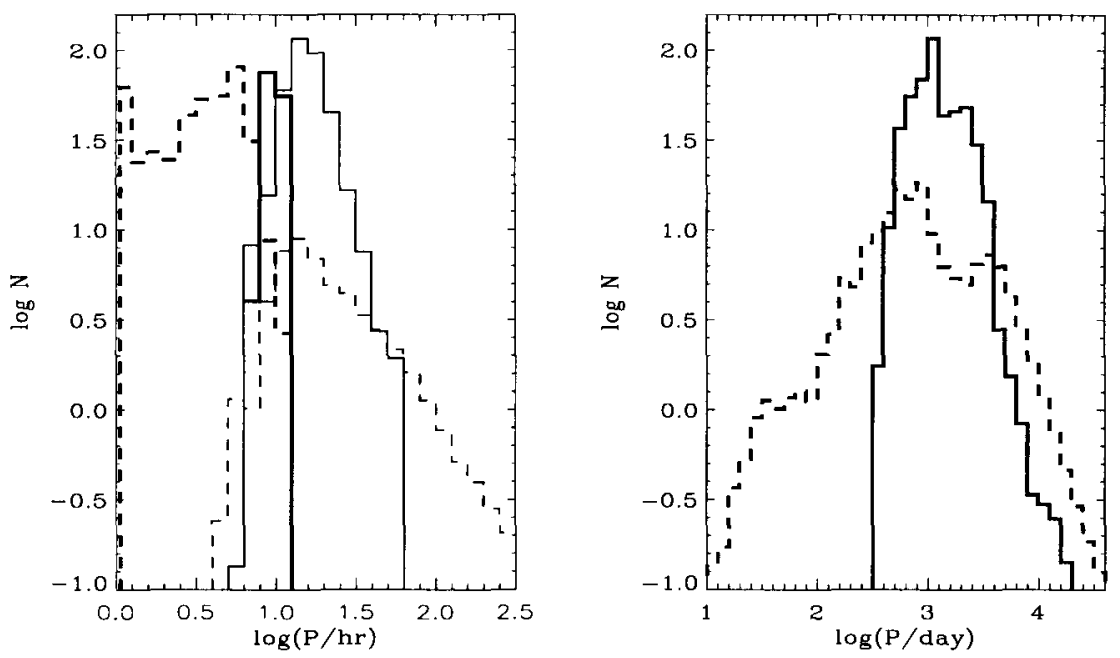

Figure 1. Distribution of SSS over orbital periods. Left panel-CVs (thick lines) and Algols (thin lines), right panel-SyS. Solid line-permanent sources, broken line-transient sources.

'transient' sources, in which supersoft X-rays are expected to be radiated for short ( $\lesssim 150 \mathrm{yr}$ ) intervals of time following shell burning flashes. The different parent groups differ in terms of the number of objects which they contain of the two classes. High accretion rates result in a high proportion of permanent sources, while the low ones produce transient SSS.

The detectability of SSS may be influenced by intrinsic absorption. For the transient SSS the most efficient source of intrinsic absorption may be the shielding by the envelopes ejected during nova eruptions. For SyS, all the permanent sources have an optical depth in the spherically symmetric donor wind of $\tau>1$ at the distance of the accretor from the donor, and only about $4 \%$ of the transient sources remain observable.

For high mass WDs, shielding times are longer than the lifetimes in the 'on' state. For example, a source with a mass exceeding $\sim 1.15 \mathrm{M}_{\odot}$ may never be observed. While a typical WD mass in observed nova systems is $\sim 1 \mathrm{M}_{\odot}$, in the model of the detectable sample of SSS it is between $0.6 \mathrm{M}_{\odot}$ and $0.8 \mathrm{M}_{\odot}$. There may be a higher chance to find a SSS in a survey of old ( $\lesssim 150$ yr) novae. This may explain why only one SSS was found in a survey of 26 novae, which exploded in the last $10 \mathrm{yr}$ (Orio 1993).

For the detection of supersoft emission, the source has to be closer than $\sim 2 \mathrm{kpc}$ to the Sun. For the identification of a transient source with a nova, we apply the requirement for the nova to have $V \leq 8 \mathrm{mag}$ in the plateau luminosity stage. Combined, intrinsic and interstellar absorption leave only 
$\sim 25$ 'detectable' Galactic SSS.

Other possible contributors to the Galactic population of SSS are semidetached, double degenerate systems with He WD donors (Tutukov \& Yungelson 1996) and semi-detached systems in which the donors have CO cores and thick helium mantles ('helium Algols'; Iben \& Tutukov 1994). In these systems mass exchange may be stable, steady He burning at the surface of WDs occur and hence, emission of supersoft X-rays is possible. Finally, planetary nebula nuclei $(\mathrm{PNNi})$ more massive than $0.7 \mathrm{M}_{\odot}$ spend a certain time at temperatures in excess of $250000 \mathrm{~K}$ and have at this time $L \gtrsim 510^{3} \mathrm{~L}_{\odot}$.

Fig. 1 shows the distribution of model SSS over $P_{\text {orb }}$. Permanent sources appear in two distinct ranges of periods with peaks close to $10 \ldots 20 \mathrm{~h}$ and $500 \ldots 3000 \mathrm{~d}$. Transient sources in CVs are evenly distributed between the 'minimum' period of CVs and $8 \mathrm{~h}$. Algols cover the period range between $10 \mathrm{~h}$ and $100 \mathrm{~h}$ (with a 'tail' to $300 \mathrm{~h}$ ), and in SyS the transient sources have $100 \lesssim P_{\text {orb }}(\mathrm{d}) \lesssim 10000$. Every class of model objects has its counterpart among the discovered Galactic SSS. For example, GQ Mus is a post-nova. system, sources RX J0925.7-4756 and RX J0019+21 may be identified with systems with subgiant donors, and the symbiotic stars RR Tel and AG Dra confirm the contribution of this class.

Depending on the secular evolution of the accretion rate and initial mass of the WD, the latter may decrease or grow. We estimate that the rate at which accreting WDs in Galactic binaries reach the Chandrasekhar mass is only $310^{-5} \mathrm{yr}^{-1}$. If the WD is not eroded by novae, a He layer accumulates, and it may detonate when the critical mass for ignition is accumulated. Helium detonation may result in the detonation of the core and a supernova (e.g. Livne \& Glasner 1990). The only model systems in which the accumulation of a critical layer for detonation of He is possible are Algols. The estimated Galactic rate of possible detonations is $310^{-4} \mathrm{yr}^{-1}$.

\section{References}

Iben, I., Tutukov, A.V., 1989, Ap. J., 342, 430

Iben, I., Tutukov, A.V., 1994, Ap. J., 431, 264

Livne, E., Glasner, A.S., 1990, Ap. J., 361, 244

Orio, M., 1993, in "Cataclysmic Variables and Related Physics", eds O. Regev, G. Shaviv, Ann. Israel Phys. Soc., 10, p219

Prialnik, D., Kovetz, A., 1995, Ap. J., 445, 789

Tutukov, A., Yungelson, L., 1996, MNRAS, in press

van den Heuvel, E.P.J., Bhattacharya, D., Nomoto, K., Rappaport, S.A., 1992, A\&A, 262, 97

Yungelson, L., Livio, M., Tutukov, A., Kenyon, S.J., 1995, Ap. J., 447, 665 